\title{
A Experiência da Monitoria de Algoritmos e Programação em Cursos de Engenharia na Perspectiva dos Monitores
}

\author{
José Solenir Lima Figuerêdo, Jussara Gomes Machado, Samuel Vitório Lima, Cláudio Sérgio da \\ Silva Cerqueira, Claudia Pinto Pereira \\ UEFS - Universidade Estadual de Feira de Santana \\ Feira de Santana, Bahia, Brasil \\ \{jslfigueredo@ecomp.uefs.br\},\{jussarauefs,samvlima10\}@gmail.com,\{csscerqueira,claudiap\}@uefs.br
}

\section{RESUMO}

A monitoria é uma modalidade de ensino e aprendizagem que contribui para a formação integrada dos estudantes nas atividades de ensino, pesquisa e extensão. O exercício da monitoria é uma oportunidade para o estudante monitor desenvolver habilidades inerentes à docência, além de aprofundar conhecimentos na área específica. No entanto, apesar da motivação otimista na concepção da monitoria, a sua ação efetiva depende de muitos fatores, que vão desde o interesse particular dos estudantes "monitorados" até às atividades desenvolvidas pelo monitor. Neste sentido, refletir e compreender como os monitores enxergam a monitoria constitui-se uma atividade fundamental. Assim, este estudo relata a experiência vivenciada pelos estudantes monitores vinculados a um projeto de monitoria que auxilia disciplinas que trabalham com tópicos relacionados à lógica e ao raciocínio matemático, notadamente a programação de computadores. Junto à experiência vivenciada, são apontadas as principais dificuldades e possíveis intervenções. Os resultados revelam que "Contribuir significativamente com o processo de aprendizagem de outros estudantes" e o "Interesse em ingressar na vida acadêmica/docente" são os principais motivos que levam os estudantes a entrarem no projeto de monitoria. Também indicaram que a monitoria permitiu melhorar suas estratégias de ensino, o conhecimento sobre linguagens de programação e a oralidade. Além disso, os monitores apontaram que a monitoria consegue ajudar, efetivamente, os alunos em relação à linguagem de programação e ao raciocínio lógico. As principais lições aprendidas foram: o aprofundamento do conhecimento, o potencial da aprendizagem colaborativa e, finalmente, a dificuldade com conceitos abstratos de computação.

\section{PALAVRAS-CHAVE}

monitoria, aprendizado ativo, aprendizagem de computação, programação de computadores

\section{INTRODUÇÃO}

Nos últimos 20 anos, o ensino superior no Brasil passou por um intenso processo de expansão. Conforme apontado pelo Instituto Nacional de Estudos e Pesquisas Educacionais Anísio Teixeira (INEP),

Fica permitido ao(s) autor(es) ou a terceiros a reprodução ou distribuição, em parte ou no todo, do material extraído dessa obra, de forma verbatim, adaptada ou remixada, bem como a criação ou produção a partir do conteúdo dessa obra, para fins não comerciais, desde que sejam atribuídos os devidos créditos à criação original, sob os termos da licença CC BY-NC 4.0.

EduComp'21, Abril 27-30, 2021, Jataí, Goiás, Brasil (On-line)

(C) 2021 Copyright mantido pelo(s) autor(es). Direitos de publicação licenciados à Sociedade Brasileira de Computação (SBC). entre 2007 e 2017 houve um aumento de 56,4\% na matrícula do ensino superior [19]. Contudo, embora se observe esse aspecto positivo no ensino superior brasileiro, alguns problemas ainda persistem, como de estrutura física, formação de professores, redução orçamentária, e, ainda, altos índices de evasão e reprovação, sendo os cursos de engenharia os mais afetados neste último caso [42].

Ao ingressarem no ensino superior, uma nova realidade é apresentada aos estudantes, considerando-se múltiplos aspectos, mas principalmente em relação às disciplinas e aos conteúdos, que muitas vezes acabam se distanciando daquilo que estavam habituados. Essa realidade é presenciada em diferentes cursos, mas sobretudo nas engenharias, com os estudantes novatos, os quais precisam se adaptar à universidade, com suas características e particularidades, como também aos novos componentes curriculares e suas diversidades [38]. Essa adaptação está longe de ser trivial, por variados motivos, tais como as experiências pessoais anteriores, as fragilidades na escolaridade anterior, as dificuldades de relacionamento, de interpretação de problemas, de raciocínio lógico matemático, de abstração e outros [29, 41]. Disciplinas que naturalmente estão envolvidas com o pensamento computacional, como a programação de computadores, são exemplos típicos de disciplinas que exigem a capacidade de interpretação e a abstração por parte dos discentes $[43,54]$.

A programação de computadores se apresenta como uma competência/habilidade complexa de se aprender, pois exige diferentes variedades de conhecimentos, estratégias e modelos nos diferentes níveis de domínio do problema, do design de solução e do programa propriamente dito [44]. Por esses e outros motivos, àquelas disciplinas que envolvem programação ou, até mesmo, o uso do pensamento computacional acabam por apresentar altos índices de abandono e reprovação [53].

Para minimizar as dificuldades já mencionadas, um série de ações podem ser empregadas, sejam elas institucionais, curriculares, docentes e discentes, tais como, monitoria, estudos individuais sistematizados ou em grupo, prática de exercícios e leituras, projetos disciplinares ou interdisciplinares, estágio supervisionado e/ou não supervisionado, organização de tempo e agenda de estudos [7]. A aplicação dessas estratégias de maneira contínua pode contribuir para reduzir as dificuldades enfrentadas pelos estudantes. Em nosso contexto, tem-se interesse particular nas atividades relacionadas à monitoria.

A monitoria pode ser compreendida como uma modalidade de ensino e aprendizagem que contribui para a formação integrada dos estudantes nas atividades de ensino, pesquisa e extensão dos cursos de graduação [32]. A monitoria tem um importante papel 
nos cursos de graduação, especialmente em razão da estreita relação existente entre o aluno monitor e os demais estudantes, o que contribui mutuamente para o desenvolvimento de ambos, devido à troca de informações que pode haver entre si. O estudante precisa do conhecimento adquirido pelo monitor para solucionar uma dúvida, e este, por sua vez, precisa desse conhecimento para ajudar a solucionar a dúvida apresentada pelo aluno [48]. Esse aspecto colaborativo da monitoria é bem peculiar comparado a outras atividades pedagógicas, pois os dois envolvidos neste processo serão "recompensados". Conforme apontado em [48], o estudante tem a oportunidade de aprender com o auxilio do monitor, enquanto o monitor poderá adquirir mais experiência e terá a oportunidade de fixar ainda mais o conteúdo. Além disso, no papel de docente, o estudante monitor pode obter uma visão holística sobre determinado assunto, podendo levá-lo a um aprofundamento daquele conteúdo ou, até mesmo, uma ressignificação.

Contudo, embora a monitoria seja uma realidade em grande parte das universidades brasileiras, além de um importante instrumento no processo de ensino e aprendizagem, ainda são incipientes os estudos sobre o tema no âmbito do ensino superior [15]. Por conseguinte, muitas oportunidades de melhorias do programa em articulação com o ensino não são bem aproveitadas ou são pouco utilizadas [1]. Dessa forma, estudos que investiguem aspectos relacionados a essa atividade são pertinentes, uma vez que podem contribuir para melhor compreensão de como a monitoria está sendo desenvolvida, contribuindo com o aprimoramento contínuo dessa atividade tão importante à excelência acadêmica.

Diante do cenário apresentado, este artigo relata a experiência vivenciada pelos estudantes monitores vinculados à monitoria de algoritmos e programação da Universidade Estadual de Feira de Santana (UEFS), apontando as principais percepções adquiridas pelos monitores ao longo do desenvolvimento do projeto de monitoria. Além disso, apresentamos as principais lições aprendidas, assim como um conjunto de possíveis estratégias pedagógicas que poderiam ser aplicadas a fim de minimizar os problemas presentes nas disciplinas contempladas pela monitoria, comumente relacionados à programação de computadores. O exercício da monitoria é uma oportunidade para o estudante desenvolver habilidades inerentes à docência, além de aprofundar conhecimentos na área específica [3], o que de certa maneira auxilia na formação de profissionais mais capacitados. Deste modo, espera-se também incentivar a adoção dessas estratégias em outros projetos de monitoria semelhantes, buscando inovar nas estratégias pedagógicas.

$\mathrm{O}$ restante deste trabalho segue estruturado da seguinte forma. A seção 2 apresenta os principais conceitos norteadores deste estudo. A seção 3 descreve os principais trabalhos relacionados. Na seção 4 , os materiais e métodos utilizados são apresentados. A seção 5 apresenta os resultados alcançados neste estudo, bem como a discussão de alguns pontos. Por fim, na seção 6, relatam-se as conclusões mais significativas obtidas a partir deste trabalho.

\section{FUNDAMENTAÇÃO TEÓRICA}

Nesta seção, aborda-se brevemente uma visão geral da monitoria acadêmica no Brasil, assim como no âmbito da instituição, na qual este estudo foi realizado. Além disso, apresentam-se as principais metodologias de aprendizagem relacionadas às práticas pedagógicas envolvidas na monitoria.

\subsection{Monitoria acadêmica no Brasil}

A monitoria acadêmica no Brasil foi inicialmente proposta através da Lei n. ${ }^{\circ}$ 5.540/68 [9], que fixa normas de organização e funcionamento do ensino superior e sua articulação com a escola média. Em seu art. 41, a lei estabelecia que: "As universidades deverão criar as funções de monitor para alunos do curso de graduação que se submeterem a provas específicas, nas quais demonstrem capacidade de desempenho em atividades técnico-didáticas de determinada disciplina. Parágrafo único. As funções de monitor deverão ser remuneradas e consideradas título para posterior ingresso em carreira de magistério superior".

Posteriormente, em 1981, a monitoria acadêmica voltou a ser citada no Decreto de $\mathrm{n}^{\circ} 85.862$ [10] (posteriormente revogado), que atribuía competência às Instituições de Ensino Superior para fixar as condições necessárias ao exercício das funções de monitoria e dá outras providências.

Mais recente, a Lei $\mathrm{n}^{\circ} 9.394$ [12], que estabelece as diretrizes e normas da educação nacional, define em seu art. 84 que: "Os discentes da educação superior poderão ser aproveitados em tarefas de ensino e pesquisa pelas respectivas instituições, exercendo funções de monitoria, de acordo com seu rendimento e seu plano de estudos".

Desde a sua concepção, a monitoria acadêmica vêm contribuindo para que as universidades obedeçam ao princípio da indissociabilidade entre o ensino, a pesquisa e a extensão, expresso no art. 207 da Constituição de 1988 [11], atuando sobre a perspectiva do ensino.

\subsection{Monitoria acadêmica na UEFS}

O Programa de Bolsa Monitoria da UEFS é regulamentada através da Resolução CONSEPE 208/2010 [51] e responsabilidade da Pró-Reitoria de Ensino de Graduação (PROGRAD). Os sujeitos envolvidos neste programa são: os monitores bolsistas, os monitores voluntários, os professores orientadores e os discentes aprendentes.

Os monitores, bolsistas e voluntários, são selecionados através de editais públicos. As seleções geralmente ocorrem em três etapas: a prova escrita, através da qual se avalia, entre outros aspectos, a capacidade de síntese e ordenação lógica do pensamento; a entrevista, momento no qual se identifica os interesses do candidato e demais aspectos relevantes à atividade de monitoria; e a prova de títulos, através da qual se avalia o desempenho acadêmico, bem como a participação do candidato em atividades extra-curriculares. Tendo sido aprovado no processo seletivo, o discente monitor assume a responsabilidade de participar ativamente das atividades planejadas pelos professores orientadores, dentre as quais são comuns: auxiliar o professor na realização de trabalhos práticos e experimentais, na preparação de material didático e em atividades de classe, laboratórios, workshops e trabalhos de campo [20].

Os professores orientadores são os responsáveis por acompanhar, orientar e avaliar as atividades desenvolvidas pelos monitores. Eles também são responsáveis por fazer a solicitação anual do projeto, especificando, entre outras coisas, sua estrutura, justificativa, objetivos, o plano e as atividades a serem desenvolvidas [20]. 
De acordo com o art. 3 da Resolução CONSEPE 208/2010, são objetivos do Programa de Bolsa Monitoria da UEFS: I. Proporcionar ao estudante de graduação, regularmente matriculado, a oportunidade de engajar-se em pleno trabalho de atividades de ensino que possibilitem o aperfeiçoamento do conhecimento e o desenvolvimento de atitudes e habilidades favoráveis à sua formação acadêmica e profissional; II. Criar condições de aperfeiçoamento teórico e desenvolvimento de habilidades relacionadas à atividade docente. III. Estabelecer práticas e experiências pedagógicas que permitam a integração dos estudantes monitores com o corpo docente, discente e técnico administrativo [20].

\subsection{Metodologias de Aprendizagem}

Semelhante ao relatado por [14] referente às escolas de ensino médio no Brasil, nas universidades se percebe um desnível de conhecimentos entre os alunos. Entre as justificativas, destacam-se as diferentes condições sociais dos alunos, condições tais que refletem principalmente na qualidade da sua formação acadêmica anterior [25][22][27]. Diante dessa realidade, torna-se necessária a implementação de projetos de intervenção que visem solucionar o problema.

O Programa de monitoria surge, nesse aspecto, como uma estratégia de intervenção que promove uma aprendizagem colaborativa $\mathrm{e}$ significativa, levando os alunos envolvidos a entenderem os problemas sob uma perspectiva diferente e assim propor formas diferentes de solucioná-los [2][24][26]. O cenário em que se aplica essa forma de intervenção (alunos dispostos a melhorar, ambiente cooperativo e interativo, desconstrução da ideia de que o professor é o único detentor do conhecimento, etc.) possibilita a aplicação de metodologias de ensino que tornam o aluno o sujeito do seu processo de aprendizagem.

2.3.1 Aprendizagem Colaborativa. A monitoria evolui de um processo cooperativo, em que individualmente os envolvidos desempenhavam suas atividades com foco em um produto final, para um processo colaborativo, no qual os envolvidos desenvolvem um processo síncrono de aprendizagem, focado na troca de experiências [14].

Para [34], a aprendizagem colaborativa apresenta vantagens sociais porque ajuda a desenvolver um sistema de apoio social para alunos e a entender a diversidade entre as pessoas em interação. A colaboração, foco dessa metodologia, aumenta a autoestima, reduz a ansiedade e, no contexto de sala de aula, desenvolve nos alunos atitudes positivas em relação ao apresentador dos conteúdos [34].

Promover uma aprendizagem colaborativa envolve tornar o aprendente o agente do processo de aprendizagem, possibilitando que este reflita criticamente sobre as atividades em todas as suas fases. Isso significa dar autonomia e responsabilidade para a construção e a reconstrução das atividades, fazendo com que os aprendentes reconheçam seu poder de escolher o que ocorrerá em seguida [14][52].

Ainda segundo [14], o processo de aprendizagem colaborativa se torna ainda mais eficaz quando o sujeito, ao se tornar agente, pode também contar com a agência do seu colega.

2.3.2 Aprendizagem Significativa. A teoria da aprendizagem significativa, proposta por David Ausubel, enfatiza a aprendizagem por meio de significados (conceitos) como a mais relevante para os seres humanos [6]. Essa forma de aprendizagem requer três importantes requisitos [50], sendo eles: a oferta de um conhecimento novo e estruturado de maneira lógica; a prévia existência de conhecimentos na estrutura cognitiva do aprendiz que possibilite uma conexão com o novo conhecimento; e a atitude de aprender e conectar seu prévio conhecimento com o que pretende absorver.

A aprendizagem significativa, quando comparada com a aprendizagem mecânica, ou memorística, apresenta vantagens tais como o enriquecimento da estrutura cognitiva do aprendiz, uma aprendizagem a médio e longo prazo e a aquisição de outras novas aprendizagens, ou a reaprendizagem [39].

A aprendizagem mecânica ocorre através da absorção literal e não substantiva do novo material, quando este não consegue interagir com algum conhecimento já existente. O esforço nessa forma de aprendizagem é menor, porém volátil, com baixo grau de retenção na aprendizagem a médio e longo prazo. Para Ausubel [4][5], esse método memorístico deve ser adotado quando o aprendente não dispõe, em sua estrutura cognitiva, de conhecimentos que facilitem uma conexão com a nova informação a ser aprendida. Em outras palavras, Ausubel defende que a aprendizagem memorística seja a primeira linha de aprendizagem de um conhecimento novo, desenlaçado, sendo este posteriormente utilizado como âncora na aprendizagem de conteúdos semelhantes.

2.3.3 Aprendizagem por pares. A aprendizagem por pares, proposta pelo professor Eric Mazur [37], prima pelo entendimento e aplicabilidade de conceitos, utilizando-se da discussão entre os alunos [16] [40]. Mazur defende que após uma aprendizagem conceitual, os alunos devem ser guiados para o desenvolvimento de habilidades que permitam a aplicação dos seus conhecimentos em situações práticas. $\mathrm{O}$ ambiente educacional passa a ter uma característica mais dinâmica e democrática, favorecendo assim uma aprendizagem autônoma e colaborativa [8].

Mazur aplicou sua metodologia no curso de Física da universidade de Harvard, dividindo o processo de aprendizagem em três etapas: a introdução dos conceitos, realizada através da leitura de materiais antes das aulas; a aplicação de aulas expositivas com a intenção de esclarecer as dúvidas, aprofundar a compreensão e criar confiança nos alunos; e a promoção de diálogos iniciados a partir de questões conceituais em um contexto problematizado [40]. Ao analisar os resultados da aplicação da metodologia, o professor Mazur observou que o nível de retenção das informações pelos alunos cresceu $40 \%$ [16].

Segundo [34], a característica colaborativa da aprendizagem por pares promove, além das habilidades de pensamento crítico, as habilidades sociais, ao favorecer o desenvolvimento de comunidades de aprendizagem, e um fortalecimento psicológico ao centralizar o ensino no aluno.

\section{TRABALHOS RELACIONADOS}

A Monitoria tem um importante papel no processo de ensino e aprendizagem, especialmente por ser uma das estratégias potencializadoras da aprendizagem significativa [46]. A monitoria, na medida em que propicia compartilhamento de saberes e a vivência de experiências interpessoais acadêmicas, inclusive entre pares, influencia diretamente na qualidade do ensino de toda instituição [15]. 
Ao longo dos anos, diferentes trabalhos foram desenvolvidos no intuito de apresentar a experiência de projetos de monitoria na universidade em que estão implantados [1, 21, 33, 35, 36]

Oliveira et al. [21], do Departamento de Graduação de Enfermagem da Universidade Federal do Alto Sertão Paraibano, publicaram, neste artigo, a experiência da monitoria durante o processo acadêmico do estudante. Um dos pontos abordados é que a monitoria proporciona, para o estudante monitor, a imersão nas habilidades da docência (e.g. o processo de ensino-aprendizagem e o aprofundamento do conhecimento), além de apoiar os discentes monitorados no melhor entendimento dos conteúdos da disciplina [21]. A partir desta experiência, a monitoria proporcionou uma melhora no relacionamento entre os discentes e os docentes e o aumento do senso de responsabilidade do monitor.

A disciplina de Sistemas Corporais, vinculada à Escola da Saúde da Universidade Potiguar (UnP), campus Mossoró, a partir de um estudo dirigido, relatou a atividade de um estudante monitor. Este estudo concluiu que a experiência da monitoria proporcionou, dentre outras coisas, o conhecimento dos júbilos e dos contratempos da docência; a alegria por estar apoiando o processo de ensinoaprendizagem das pessoas e também a frustração pelo desestímulo de alguns estudantes que não queriam se esforçar para estudar um determinado conteúdo [36]. O autor identificou o aprimoramento dos conhecimentos do monitor relacionados aos conteúdos programáticos e o desenvolvimento de atividades junto com os docentes, estimulando, dessa maneira, o ingresso deste estudante na área acadêmica [36].

A disciplina de Cuidados Críticos, do Bacharelado de Enfermagem da Universidade Federal de Campina Grande, ofertou monitoria como apoio aos conhecimentos práticos e teóricos. Um artigo foi gerado para descrever como foi executado o processo de monitoria da disciplina. O monitor tinha como função a fabricação de exemplos práticos que eram aplicados nos laboratórios, permitindo uma troca de conhecimento entre o monitor e os outros alunos [35]. A partir destas atividades, percebeu-se que, além de proporcionar conhecimento na área acadêmica, a monitoria também pôde ajudar na execução de um processo que o aluno terá que realizar no mercado de trabalho [35].

A Universidade Estadual da Paraíba apresentou um estudo descritivo, do tipo relato de experiência, realizado a partir da vivência discente na monitoria das disciplinas "Anatomia Humana" e "Biologia Celular". Foi relatado que a monitoria permite que o monitor desenvolva algumas habilidades sociais e intelectuais que são importantes para o ensino [33]. Outra particularidade também apresentada neste estudo foi que alguns docentes da instituição tinham problemas na sua metodologia de ensino, e que uma das possíveis causas para isto seja o fato destes docentes não terem tido a oportunidade de participar de atividades pedagógicas em seu processo de formação [33].

Uma outra questão apontada por Lira et al. [33] é que alguns alunos podem se sentir intimidados de tirarem suas dúvidas na aula, por entenderem que a dúvida pode inferiorizar o seu conhecimento diante do professor. Neste sentido, a monitoria pode ajudá-los a sanar suas dúvidas, e diminuir a timidez e a distância, pelo fato de o monitor ser também um estudante.
Amato e Reis [1], considerando a monitoria como uma estratégia fundamental à aprendizagem significativa na medida em que possibilita a construção de saberes e a troca entre pares, desenvolveram um estudo de caso sobre o Programa de Monitoria do CEFET/RJ do curso de Engenharia de Produção. O objetivo principal do estudo era apresentar a percepção dos estudantes monitores em relação ao programa de monitoria. Os resultados obtidos pelos autores revelaram que a maioria dos monitores do CEFET/RJ ingressam no programa da monitoria em razão da possibilidade de exercer uma atividade extraclasse. Além disso, os autores identificaram que, embora o programa proporcione inúmeros benefícios à formação acadêmica e pessoal dos estudantes monitorados, ainda não se conseguiu popularizá-lo entre os discentes.

O presente estudo, de maneira similar aos já mencionados, relata a experiência vivenciada pelos estudantes monitores em um projeto de monitoria. No entanto, comparado àqueles, apresenta contribuições e inovações significativas. Diferentemente dos trabalhos mencionados, realizados em sua maioria em cursos da área de saúde, o nosso se desenvolve em disciplinas de cursos de Engenharia, sob a ótica dos monitores. Além disso, propõe-se, à luz das percepções adquiridas ao longo dos anos, um conjunto de estratégias pedagógicas que poderiam ser empregadas para contribuir com o aperfeiçoamento das disciplinas e, portanto, com o processo de aprendizagem dos discentes.

\section{MATERIAIS E MÉTODOS}

Nesta seção, apresentam-se os materiais e métodos que contribuíram para a execução desse trabalho. Inicialmente é retratada uma visão geral do ambiente no qual este estudo aconteceu e, em seguida, abordamos o processo metodológico, que inclui todas as etapas utilizadas na condução do estudo.

\subsection{Projeto de Monitoria}

O relato de experiência descrito neste estudo se deu no âmbito do projeto "Práticas Pedagógicas que Contemplam a Formação Acadêmica e Profissional dos Estudantes de Engenharia da UEFS". O projeto busca auxiliar estudantes em disciplinas que envolve a resolução de problemas e de raciocínio lógico matemático, bem como pensamento computacional. Em sua maioria, essas disciplinas envolvem a programação de computadores. Os monitores alocados no projeto possuíam, entre outras atribuições, a responsabilidade de tirar dúvidas dos discentes monitorados. Vale ressaltar que o papel do monitor, em hipótese alguma, é fazer pelo estudante, mas colaborar no sentido de ajudá-lo a progredir nos seus estudos.

Considerando os estudantes dos cursos de Engenharia, observase a ênfase e a necessidade de que esses estudantes desenvolvam a capacidade de resolução de problemas e de raciocínio lógico matemático, habilidades desejadas e trabalhadas pelos componentes curriculares "Algoritmos e Programação I" e "MI-Algoritmos", do curso de Engenharia de Computação, e "Introdução à Ciência da Computação", dos cursos de Engenharia de Alimentos e Engenharia Civil. Neste sentido, os serviços da monitoria eram oferecidos a estudantes destes cursos. De maneira especial, em razão de apresentar conceitos básicos relacionados à computação, bem como por priorizar o desenvolvimento do pensamento computacional e do 
raciocínio lógico matemático dos estudantes, o componente curricular "Informática Aplicada" dos cursos de Química e Agronomia também fez parte do rol de disciplinas apoiadas pela monitoria.

Para o desenvolvimento das atividades da monitoria, $12 \mathrm{~h}$ semanais eram reservadas, preferencialmente em turnos opostos aos das aulas, a fim de atingir a maioria dos estudantes. Ao monitor era atribuído o papel de acompanhar os estudantes em alguns momentos/horários no laboratório, ajudando-os a compreender e utilizar o ambiente de desenvolvimento, a linguagem de programação e os conteúdos estudados, em especial, nas práticas das disciplinas envolvidas. Para tanto, ao longo dos anos da monitoria, diferentes estratégias foram empregadas a fim de contemplar esse aspecto, entre as principais estão:

- Encontros presenciais com os estudantes: Horários semanais eram reservados para encontros com os discentes. Qualquer aluno monitorado poderia participar desses encontros para tratar sobre as suas dúvidas. As dúvidas eram resolvidas por meio de exemplos práticos, a partir da utilização de questões semelhantes às que eram mencionadas pelos discentes, teste de mesa ou a partir de representações gráficas para o melhor entendimento da estrutura, este último caso sendo mais utilizado na explicação de estruturas de dados como listas encadeadas e matrizes.

- Atendimento utilizando recursos digitais: Além dos encontros presenciais da monitoria, os estudantes monitorados tinham a oportunidade de tirar dúvidas com os monitores por outros meios de comunicação, como e-mail, WhatsApp, Discord, entre outras. Essas ferramentas eram úteis principalmente para àqueles estudantes que não residiam próximo da Universidade, o que acrescentaria mais um empecilho em buscar a monitoria. Além disso, poderia ocorrer incompatibilidade do horário estabelecido pelo monitor para atendimento presencial com aqueles de disponibilidade do discente. Assim, esses recursos se tornavam uma alternativa para estudantes que se enquadravam nesse perfil.

- Participação em aulas de exercícios das disciplinas teóricas: Em determinado período, as disciplinas teóricas, com as quais a monitora colaborava, realizavam aulas de exercícios, notadamente antes de avaliações. Em algumas oportunidades, os monitores também participaram dessas aulas, ajudando àqueles estudantes que apresentavam dúvidas. Era um momento de construção colaborativa (i.e. professores, estudantes e monitores) de soluções para os problemas/exercícios apresentados.

- Produção de materiais didáticos: Além dos encontros presenciais e a utilização de outros recursos para aprimorar o serviço prestado, os monitores também desenvolviam materiais pedagógicos para apoiar os estudantes. Foram produzidas listas de exercícios, que eram então disponibilizadas no site vinculado à monitoria ${ }^{1}$, assim como vídeo aulas e apostilas das temáticas vistas em aula (e.g. operadores lógicos e aritméticos, estruturas condicionais, estruturas de repetição, estruturas de dados, funções, entre outras).

- Aplicação de minicursos preparatórios: Durante a semana de integração do curso de Engenharia de Computação,

\footnotetext{
${ }^{1}$ http://monitoriaalgoritmos.uefs.br/
}

que acontece geralmente na primeira semana de aulas, estudantes monitores aplicam um minicurso abordando de forma lúdica assuntos relacionados à lógica, algoritmos e programação. Tais minicursos têm como objetivo promover, nos estudantes novatos, o desenvolvimento de um conhecimento inicial que posteriormente favoreça uma aprendizagem significativa. Os minicursos aplicados são comumente abordados em três etapas: (1) Fluxograma Humano, focado na aprendizagem de algoritmos por meio da computação desplugada; (2) Jogo de Lógica, que trata da aprendizagem por meio da colaboração e da divisão de tarefas, e; (3) Introdução a Programação, transformando algoritmos em códigos executáveis por meios de comandos básicos da linguagem de programação C e/ou Python.

As atividades mencionadas tencionavam contribuir no processo de aprendizagem dos estudantes monitorados, assim como na inserção do monitor à prática docente. Durante a aplicação dessas atividades e dos atendimentos realizados, os monitores têm a oportunidade de realizar observações e adquirir novas percepções da prática docente, especialmente relacionadas ao ensino de programação de computadores. É neste contexto, sob observações e percepções, que esse trabalho se desenvolve. Mais detalhes acerca do processo metodológico são apresentados em sequência.

\subsection{Processo Metodológico}

O presente estudo trata-se de um relato de experiência que visa descrever as vivências de estudantes, enquanto monitores de um projeto de monitoria. Metodologicamente, relatos de experiências permitem realizar a descrição de experiências vivenciadas, de natureza qualitativa, tendo em vista que evidenciam aspectos subjetivos do ser humano [28, 47]. Neste sentido, por meio do relato de experiência, é possível suscitar novos debates e reflexões sobre uma determinada temática.

Desde a sua implantação, em 2016, até o presente momento, a monitoria já contou com a participação de 9 estudantes monitores, todos discentes do curso de Engenharia de Computação. $\mathrm{O}$ fato de todos os monitores serem oriundos do curso de Engenharia de Computação justifica-se por conta da natureza das disciplinas contempladas pelo projeto, as quais em geral requerem competências de programação de computadores, com ênfase na resolução de problemas. Assim, este estudo foi realizado com base nas experiências desses estudantes monitores, com relação às atividades didáticopedagógicas efetuadas no período que estiveram na condição de monitor.

A condução deste estudo seguiu basicamente 5 etapas, indicadas na Figura 1. Inicialmente foi definida a temática a ser abordada, com auxilio dos três últimos monitores envolvidos com o projeto, os quais figuram entre os autores deste trabalho. Em uma outra etapa, foi realizado um levantamento bibliográfico, no intuito de encontrar os principais conteúdos associados à monitoria, bem como trabalhos semelhantes ao abordado neste artigo. Em seguida, elaborou-se um questionário ${ }^{2}$ para o levantamento de dados, composto tanto por questões abertas quanto fechadas. Em um outro momento, aplicou-se o questionário com os monitores que fizeram

${ }^{2} \mathrm{O}$ questionário pode ser encontrado em: https://forms.gle/XJ29Sw8pqDmH8t7F6 
parte do projeto de monitoria até a data deste estudo. O questionário tinha como objetivo avaliar a experiência dos monitores que participaram da monitoria ao longo dos anos. Com isso, buscava-se entender de que forma a participação na monitoria contribuiu com o aprimoramento pessoal e técnico desses estudantes. Além disso, buscava-se fazer um levantamento das principais lições aprendidas, incluindo as dificuldades percebidas e as habilidades adquiridas durante o período que participaram do projeto da monitoria. Por fim, na quinta etapa, fez-se a análise dos dados coletados. Vale salientar que apesar da utilização de questionários para a coleta de dados, esse estudo ainda assim mantém um caráter qualitativo, pois está centrado nos aspectos subjetivos [30].

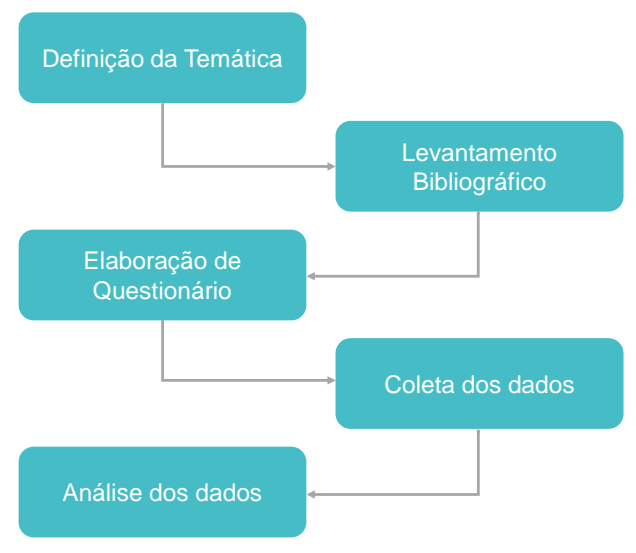

Figura 1: Workflow de desenvolvimento do estudo.

\section{RESULTADOS E DISCUSSÃO}

Os resultados estão separados em três seções principais. Na primeira seção, realiza-se uma análise dos resultados obtidos por meio dos questionários. Busca-se ilustrar os principais pontos indicados pelos monitores. Enquanto isso, na segunda seção, abordam-se as principais percepções e dificuldades encontradas durante o desenvolvimento da monitoria. Por fim, na última seção é elencado um conjunto de lições aprendidas pelos monitores ao longo do projeto de monitoria, que foram descritas no questionário. Ainda na seção das lições aprendidas relacionadas a desafios/problemas, sugere-se um conjunto de possíveis intervenções, como forma de minimizá-los. Com essas sugestões, se aplicadas, espera-se que haja um aprimoramento no processo de ensino/aprendizagem do discente.

\subsection{Experiência dos monitores}

A inscrição no projeto de monitoria pode estar associada a diferentes motivações, sejam de cunho intrínseco ou extrínseco. Considera-se como motivação intrínseca aquela na qual o individuo busca, naturalmente, novidades e desafios, não sendo necessárias pressões externas ou prêmios pelo cumprimento da tarefa, uma vez que a participação nessa é a recompensa principal [17]. A motivação extrínseca, por sua vez, é aquela vinculada a algo externo à tarefa ou atividade, como, por exemplo, para a obtenção de recompensas materiais, sociais ou de reconhecimento [17]. A Figura 2 exibe os principais motivos que levaram os monitores a participarem do projeto de monitoria. Como pode ser observado, os principais motivos indicados pelos estudantes foram "Contribuir significativamente com o processo de aprendizagem de outros estudantes" (100\%), o "Interesse em ingressar na vida acadêmica/docente" (88, 7\%) e "Aprimorar minhas habilidades relacionadas à programação de Computadores" (66,7\%). De modo geral, as escolhas realizadas pelos estudantes corroboram com os objetivos que sustentam a criação de projetos de monitoria. Afinal, entre vários objetivos que justificam a criação da monitoria está a busca por ser uma ferramenta de importância no processo de aprendizagem dos estudantes, além de ser um passo inicial de iniciação à docência [3].

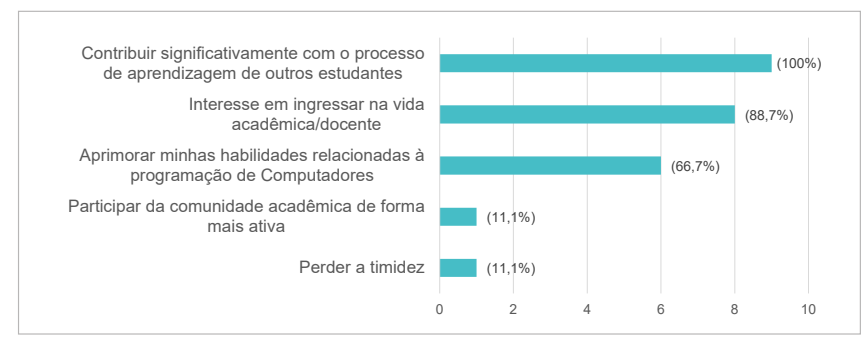

Figura 2: Motivos principais de ingresso na monitoria.

A Figura 3 ilustra como foi o grau de experiência do monitor, considerando o período deste nesta condição. Observou-se que, em sua grande maioria, os monitores indicaram a experiência como Muito Bom (56\%). Outros indicaram como sendo Excelente (33\%) e Bom (11\%). Ressalta-se que nenhum dos monitores que responderam o questionário apontou a experiência como Razoável ou até mesmo Ruim. Esse resultado mostra que a monitoria tem sido uma experiência positiva para os discentes que dela participam, mesmo diante de dificuldades inerentes ao processo.

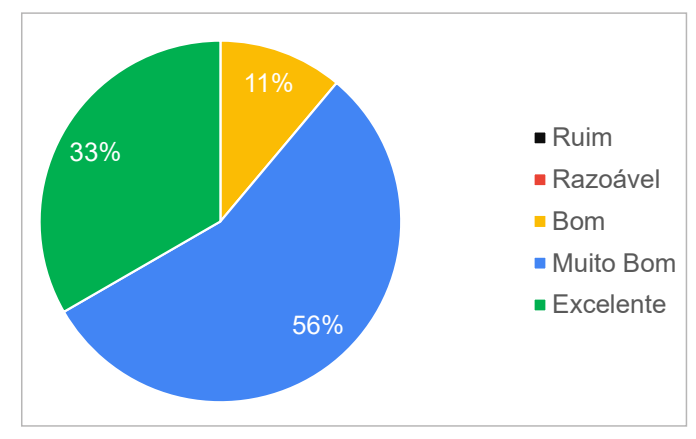

Figura 3: Experiência como monitor no projeto da monitoria.

Com relação aos cursos de Engenharia, o Conselho Nacional de Educação [18] prevê que o engenheiro dos cursos de graduação deve ter "formação generalista, humanista, crítica e reflexiva, capacitado a absorver e desenvolver novas tecnologias, com atuação crítica e 
criativa na identificação e resolução de problemas”. Diante disso, buscou-se avaliar algumas dessas dimensões, como forma de verificar o impacto da monitoria em alguns desses aspectos. Assim, na Figura 4, exibe-se uma relação das habilidades/competências aprimoradas a partir da participação na monitoria. Conforme indicado pelos participantes, características como Metodologia de ensino (100\%), Relação interpessoal (88, 9\%), Empatia (77, 8\%), Oralidade $(77,8 \%)$ e Responsabilidade $(66,7 \%)$ tiveram um impacto positivo. De modo geral, percebe-se que a monitoria contribuiu com o processo de formação dos discentes para se alcançar alguns dos objetivos definidos pelo CNE, tão importantes para os engenheiros.

A monitoria, em vários aspectos, pode contribuir na formação dos estudantes. O resultado revelado na Figura 4 ratifica a importância de uma formação ampla para os estudantes de engenharia. Afinal, o processo de formação e qualificação do engenheiro, de maneira similar a outros profissionais, requer um arcabouço de conhecimento diferenciado, aliado à capacidade de análise em profundidade da realidade política, social, cultural, entre outras habilidades exigidas para viver e conviver no mundo moderno [13]. Vale ressaltar que esse processo formativo deve levar em conta as necessidades da nação e o bem-estar da sociedade, como apontado em Cordeiro et al. [13].

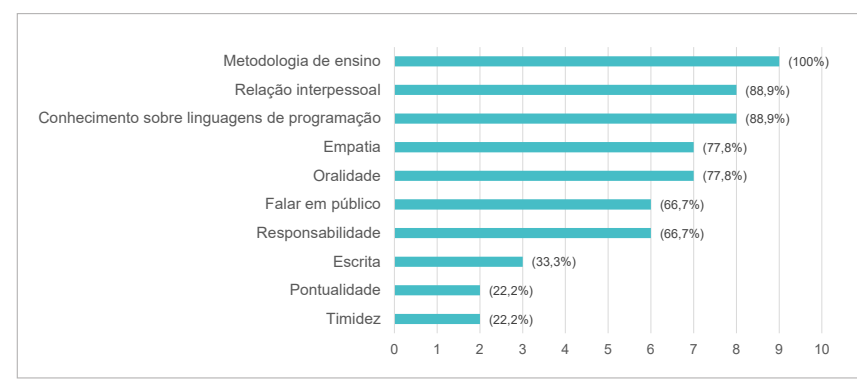

Figura 4: Habilidades/competências aprimoradas a partir da participação na monitoria.

Considerando a efetividade da monitoria com relação à linguagem de programação e ao raciocínio lógico, os monitores indicaram assertivamente a sua eficácia. Essa percepção da efetividade foi relatada por todos os monitores (100\%). Isso indica, entre outras coisas, que a monitoria contribuiu com o processo de aprendizagem dos estudantes monitorados, tomando como visão a perspectiva dos monitores. Vale salientar que uma investigação mais aprofundada, realizada diretamente com o estudantes monitorados deve ser conduzida, para então se chegar a conclusões mais efetivas.

\subsection{Percepções e dificuldades encontradas na monitoria}

A monitoria pode contribuir positivamente em diferentes aspectos para o monitor, sejam pessoais e/ou intelectuais. A relação de troca de conhecimentos proporcionada pela monitoria se revela importante para o desenvolvimento interpessoal dos envolvidos.

Analisando o conteúdo do questionário, encontram-se relatos de como a monitoria impactou em habilidades interpessoais, especialmente com relação a habilidade de falar com pessoas, uma vez que as atividades da monitoria exigem constante exposição. Alguns dos monitores perceberam a importância do ensino no processo de aprendizagem, isto é, aprender ensinando. Todos esses aspectos de alguma forma estão relacionados com princípios defendidos pela aprendizagem colaborativa e em pares, na medida em que põem os estudantes como os principais agentes do seu aprendizado, primando pela autonomia, responsabilidade e troca de experiência entre os envolvidos [14, 37]. De maneira particular, também corrobora com o saudoso Paulo Freire, quando ele afirma que "Quem ensina aprende ao ensinar e quem aprende ensina ao aprender" [23]. Isso revela a importância da monitoria no processo de ensino/aprendizagem dos discentes, pautado em uma educação que os põem como protagonistas do seu aprendizado. Outros mencionaram a importância de introduzir aplicações de conteúdos de forma lúdica para explicar assuntos potencialmente complexos.

Considerando as dificuldades encontradas enquanto monitores do projeto, cinco, dos nove respondentes, relataram ter percebido uma resistência dos alunos em procurar a monitoria. Entre as possíveis causas foram citados: problemas relacionados ao transporte; dificuldade de acesso à universidade em horários fora das aulas; falta de tempo devido a outras atividades acadêmicas; e a estranheza à ideia de monitoria.

Ainda considerando as dificuldades, quatro dos monitores mencionaram dificuldades relacionadas aos laboratórios e salas utilizadas. Entre os problemas citados estão: a dificuldade de acesso aos locais da atividade; a falta de recursos, tais como quadros, pincéis, computadores e internet; e o tamanho dos espaços utilizados. Estas questões podem acabar afetando o desenvolvimento do projeto da monitoria, tendo em vista o desgaste que isso pode gerar.

Com relação aos alunos monitorados, relatos individuais mencionam a resistência dos alunos à aplicação de metodologias colaborativas de aprendizagem; falta de gerenciamento do tempo para poder participar dos encontros da monitoria; falta de motivação em relação às atividades propostas; dificuldades em conciliar as atividades acadêmicas com as atividades de monitoria; e uso da monitoria para resolver de forma fácil as atividades avaliativas passadas pelos professores em sala.

\subsection{Lições aprendidas}

O presente trabalho se configura, fundamentalmente, como um relato de experiência. Neste sentido, registram-se algumas lições mais importantes aprendidas pelos monitores ao longo dos anos com o projeto de monitoria.

Aprofundamento do conhecimento. A experiência com a monitoria proporcionou muitos ganhos pessoais para os monitores, como empatia, oralidade e relação interpessoal. Além desses aspectos, a monitoria pôde contribuir com o aprofundamento dos conteúdos de computação que eram abordados, uma vez que era necessário fazer uma revisão do conteúdo, visando a oferta de um atendimento cada vez mais qualificado aos discentes monitorados. Além disso, como os monitores geralmente são estudantes que já estão um pouco mais avançados no curso, rever o conteúdo depois de já ter cursado disciplinas mais avançadas do curso permitiu enxergar o tópico por outro ângulo e compreender melhor o seu funcionamento. 
Aprimoramento de competências/habilidades interpessoais. A experiência da monitoria proporcionou o aperfeiçoamento de competências interpessoais, tão importantes nos dias atuais. Habilidade como o processo de ensinar, especialmente no que diz respeito à organização e à estruturação dos conteúdos e na forma de repassar o conhecimento, foi uma delas. Ademais, houve um aprimoramento em relação à capacidade de ouvir, de desenvolver o raciocínio em uma conversa e a construção de laços de amizade. Também houve um avanço em habilidades como a oratória e a capacidade de trabalhar em equipe, assim como desenvolvimento da inteligência emocional.

Aprendizagem colaborativa. A partir da experiência da monitoria, percebeu-se como a aprendizagem colaborativa é um tema atual e de significativa importância para os professores e estudantes, sendo um dos fatores mais presentes na monitoria, especialmente quando se fala em aprendizado em pares. No entanto, percebeu-se também que esse tipo de aprendizagem ainda apresenta certa resistência por parte dos estudantes monitorados, possivelmente devido a fatores como timidez e dificuldade de compartilhar o conhecimento (medo de perda de controle). No entanto, apesar da presença dessas dificuldades e resistências em sua aplicação, utilizar a aprendizagem colaborativa resulta em bons resultados, especialmente se associado com estratégias lúdicas, uma vez que se aproveita dos melhores aspectos que ambas estratégias podem promover. Ao aplicar a aprendizagem colaborativa junto a estratégias lúdicas, pode-se promover uma sinergia, que inclui um esforço contínuo de colaboração entre os alunos, potencializando a troca de experiências e consequente aprendizagem [14]. As atividades lúdicas podem motivar enquanto divertem e aumentar a capacidade de retenção do que é ensinado, a exemplo de jogos educacionais [49].

Ensino personalizado. Cada estudante possui uma forma diferenciada de lidar com a Universidade, seja em razão de experiências pessoais anteriores ou, até mesmo, pelas fragilidades na escolaridade anterior. Em função dessas características, junto às dificuldades de interpretação de problemas e de raciocínio lógico matemático, muitas vezes fez-se necessário adaptar o discurso para individualizar o atendimento, conforme o perfil do estudante.

Observou-se a importância de personalizar o ensino (e.g. uso de exemplos contextualizados, escuta ativa/passiva, execução de código passo a passo, uso frequente de analogias, exercícios com níveis de dificuldades intercalados, uso do quadro para o estudante monitorado explicar algum conteúdo, sessão de brainstorm, entre outras.), tendo em vista o grau de experiência de cada estudante. Tudo isso, em certa medida, tem forte relação com a ideia de aprendizagem significativa defendida por Ausubel, especialmente com relação a construção espontânea de novos conceitos com base em seu conhecimento prévio, incorporando inúmeras vantagens quando comparada com a aprendizagem mecânica, ou memorística [39].

Motivação. Muitos dos alunos que procuraram a monitoria relatam situações pessoais que sugerem a presença de problemas motivacionais, o que pode acabar afetando o seu rendimento acadêmico [45], ocasionando outros problemas que comumente afetam os cursos de engenharia, como reprovações e evasão [42]. Neste sentido, percebeu-se que o monitor, além de ensinar assuntos técnicos de programação, também deve estar atento aos aspectos motivacionais dos estudantes para que, na medida do possível, tente motiválos, inserindo práticas pedagógicas que despertem a motivação, o engajamento e aumentem a confiança desses estudantes. Aqui vale ressaltar a relação existente entre a personalização do conteúdo com a motivação. A busca pela personalização do ensino é um dos caminhos que pode ser trilhado em uma tentativa de despertar a motivação dos estudantes monitorados, especialmente a motivação intrínseca.

Dificuldade com conceitos abstratos de computação. A vivência direta com os estudantes permitiu perceber que determinados conceitos, potencialmente abstratos de programação de computadores, acabam gerando inúmeras dúvidas nos alunos. Em geral, conceitos como estruturas matriciais, alocação de memória, métodos de ordenação e recursividades geraram dúvidas recorrentes nos discentes.

\subsection{Intervenções pedagógicas}

Ao longo do desenvolvimento da monitoria, diferentes problemas foram percebidos como as dificuldades já mencionadas. Para minimizar esses e outros problemas, algumas estratégias intervencionistas podem ser empregadas, como por exemplo:

- Inclusão de atividades lúdicas: Atividades lúdicas poderiam ser utilizadas de forma mais efetiva, especialmente para explicar àqueles conceitos considerados potencialmente abstratos como estrutura de repetição, estruturas matriciais, alocação de memória, métodos de ordenação e recursividades. Para apoiar essa intervenção, ambientes como o scratch e o code.org poderiam ser utilizados. Além disso, a computação desplugada também poderia ser empregada em conjunto com outras estratégias.

- Intensificar a utilização de metodologias ativas de ensino: Embora a monitoria em si já possua aspectos de metodologias ativas, como a aprendizagem em pares, é preciso intensificar a utilização de estratégias que promovam o protagonismo do estudante em seu processo de aprendizagem. Deste modo, com o apoio dos professores das disciplinas contempladas pela monitoria, poderiam ser utilizadas estratégias como sala de aula invertida, gamificação e pequenos projetos para serem resolvidos em grupo em sala de aula. Além disso, quando possível, convidar os estudantes para explicar alguns conteúdos após estudo anterior. Com relação a esta última parte, esse convite poderia ser realizado de forma aleatória, por meio de sorteio por exemplo, de modo que todos os estudantes se preparassem para executar tal atividade.

- Inserir atividades que despertem motivação: Como indicado em Santana et al. [45], a motivação afeta, direta ou indiretamente, o desempenho do estudante, com consequências explícitas no processo de aprendizagem. Neste sentido, uma alternativa que poderia ser utilizada para minimizar ou, até mesmo, superar este problema é partir da incorporação de estratégias que despertem a motivação desses estudantes, tais como: utilização de jogos; promover aulas interativas; incentivar o aluno a chegar a resposta sem entregar a resposta de imediato (que já vem sendo feito); atividades com objetivos bem definidos, ou seja, objetivos claros e divididos em etapas menores; e atividades que permitam aos estudantes perceberem a utilidade do assunto, ou seja, relacionado a uma aplicação prática de conhecimento dos discentes [45] 
ou a conhecimentos prévios e contextualizados. Para tanto, poderia ser empregado o ensino de programação em Python com mídias, utilizando áudio e imagens por exemplo, a partir do ambiente de desenvolvimento JES [31], do inglês fython Environment for Students.

As intervenções supracitadas podem ser incorporadas em diferentes ambientes, sejam nas aulas das disciplinas apoiadas pela monitoria, como nos encontros individualizados realizados pelo monitor. Para esses momentos, seria importante priorizar àqueles assuntos considerados mais difíceis de aprender, tomando como base o que é dito pela literatura, assim como a própria experiência dos professores que ministram as aulas, cujo nível de experiência permite perceber as maiores dificuldades dos discentes.

\section{CONCLUSÃO}

O presente estudo relata a experiência vivenciada na monitoria de Algoritmos e Programação da Universidade Estadual de Feira de Santana, sob a ótica dos estudantes monitores. Além disso, elenca-se um conjunto de estratégias pedagógicas que poderiam ser empregadas para contribuir com o aperfeiçoamento das disciplinas apoiadas pelo programa de monitoria. Os resultados revelam que os monitores têm como maior motivação para o ingresso no programa a possibilidade de "Contribuir significativamente com o processo de aprendizagem de outros estudantes" e o "Interesse em ingressar na vida acadêmica/docente". Isso indica, entre outras coisas, o interesse dos estudantes por um aprendizado significativo, incluindo no seu processo formativo atividades dinâmicas. Os resultados também apontam, à luz dos relatos dos próprios monitores, que houve uma aprimoramento na sua forma de ensinar, no seu conhecimento sobre linguagens de programação e na oralidade.

Outros resultados revelam a importância do programa de monitoria no processo de ensino/aprendizagem, especialmente com relação à linguagem de programação e ao raciocínio lógico. No entanto, verificou-se que a ideia da monitoria ainda enfrenta resistência pelos discentes da instituição, evidenciado especialmente pelo fato da busca pela monitoria durante o período letivo não ocorrer com muita frequência. Isso exige, sobretudo por parte da Universidade, maior promoção dos programas de monitoria, em um processo constante de divulgação e de conscientização dos discentes da importância destas iniciativas no processo de aprendizagem. Como lições aprendidas, foram apontadas pelos monitores: o aprofundamento do conhecimento, o potencial da aprendizagem colaborativa e, finalmente, a dificuldade com conceitos abstratos de computação.

O desenvolvimento deste estudo também possibilitou a proposição de possíveis estratégias visando a melhoria do programa de monitoria. Entre essas estratégias estão: inclusão de atividades lúdicas; intensificação da utilização de metodologias ativas de ensino; e inserção de atividades que despertem motivação. Com essas estratégias, espera-se contribuir de forma mais efetiva no processo de aprendizagem e no engajamento destes discentes.

Como trabalhos futuros, pretende-se estender este estudo para outras pessoas envolvidas com a monitoria, como os professores das disciplinas apoiadas pela monitoria e os discentes monitorados, a fim de verificar a percepção destes com relação ao programa, o que permitiria obter uma visão mais ampla desta iniciativa, não se restringindo somente à percepção dos estudantes monitores.
Além disso, pretende-se fazer um estudo aprofundado do impacto da monitoria no processo de ensino/aprendizagem, especialmente com relação à evasão e reprovação. Por fim, planeja-se colocar em prática as estratégias propostas neste estudo e, em seguida, avaliar o seu impacto.

\section{AGRADECIMENTOS}

Agradecemos a contribuição de todos os monitores que já foram bolsistas e voluntários do projeto de monitoria, os quais foram essenciais para o desenvolvimento deste trabalho. Também agradecemos a Pró-Reitoria de Graduação (PROGRAD) pelo apoio e suporte fornecido.

\section{REFERÊNCIAS}

[1] Danila Tavares Amato and Augusto da Cunha Reis. 2016. A percepção dos monitores sobre o programa de monitoria do Ensino Superior do CEFET/RJ. Scientia Plena 12, 7 (2016).

[2] Roberta Araújo and Lúcio Flávio Nunes Moreira. 2005. Monitoria da disciplina de Cálculo. In Congresso brasileiro de ensino de engenharia, XXXIII.

[3] Fernanda de Assis, Alessandra Zanei Borsatto, Pâmela Duarte Dias da Silva, Patrícia de Lima Peres, Patrícia Rodrigues Rocha, and Gertrudes Teixeira Lopes. 2006. Programa de monitoria acadêmica: percepções de monitores e orientadores. Rev. enferm. UER7 (2006), 391-397.

[4] David P Ausubel. 2003. Aquisição e retenção de conhecimentos: uma perspectiva cognitiva. Lisboa: Plátano 1 (2003).

[5] David Paul Ausubel, Joseph D Novak, and Helen Hanesian. 1980. Psicologia educacional. Interamericana.

[6] David Paul Ausubel, Joseph Donald Novak, Helen Hanesian, et al. 1968. Educational psychology: A cognitive view. (1968).

[7] Walter Antonio Bazzo and Luiz Teixeira do Vale Pereira. 2010. Introdução à engenharia: conceitos, ferramentas e comportamentos. Ed. da UFSC.

[8] Maria Luiza Belloni and Nilza Godoy Gomes. 2008. Infância, mídias e aprendizagem: autodidaxia e colaboração. Educação \& Sociedade 29, 104 (2008), 717-746.

[9] Brasil. 1968. LEI n ${ }^{\circ}$ 5.540, de 28 de novembro 1968. Diário Oficial da República Federativa do Brasil (1968).

[10] Brasil. 1981. Decreto ${ }^{\circ}$ 85.862, de 31 de março de 1981. Diário Oficial da União (1981).

[11] Brasil. 1988. Constituição da República Federativa do Brasil de 1988. Brasília, DF: Senado Federal. (1988).

[12] Brasil. 1996. LEI n ${ }^{\circ}$ 9.394, de 20 de dezembro 1996. Diário Oficial da República Federativa do Brasil (1996).

[13] Jõao Sérgio Cordeiro, Nival Nunes Almeida, Mario Neto Borges, Silvia Costa Dutra, Zacarias Martin Chamberlain Pravia, et al. 2009. Um futuro para a educação em engenharia no Brasil: desafios e oportunidades. Revista de Ensino de Engenharia 27, 3 (2009).

[14] Fernando Rezende da Cunha Júnior. 2017. Atividades de monitoria: uma possibilidade para o desenvolvimento da sala de aula. Educação e Pesquisa 43, 3 (2017), 681-694.

[15] Rosineide Nascimento da Silva and Maria Lusia Morais de Belo. 2012. Experiências e reflexões de monitoria: contribuição ao ensino-aprendizagem. Scientia Plena 8 , 7 (2012).

[16] Antonio Sávio dA SilvA Pinto, Marcilene Rodrigues Pereira Bueno, Maria Aparecida Félix do Amaral, Milena Zampieri Sellmann, Sônia Maria Ferreira Koehler, et al. 2012. Inovação Didática-Projeto de Reflexão e Aplicação de Metodologias Ativas de Aprendizagem no Ensino Superior: uma experiência com "peer instruction". Fanus 9, 15 (2012).

[17] Selma de Cássia Martinelli and Daniel Bartholomeu. 2007. Escala de motivação acadêmica: uma medida de motivação extrínseca e intrínseca. Avaliaçao Psicologica: Interamerican fournal of Psychological Assessment 6, 1 (2007), 21-31.

[18] Conselho Nacional de Educação (CNE). [n.d.]. Institui Diretrizes Curriculares Nacionais do Curso de Graduação em Engenharia. Resolução CNE/CES 11, de 11 de março de 2002. http://portal.mec.gov.br/cne/arquivos/pdf/CES112002.pdf

[19] Instituto Nacional de Estudos e Pesquisas Educacionais Anísio Teixeira. [n.d.]. Censo da Educação Superior: notas Estatísticas 2017. http://download.inep.gov.br/educacao_superior/censo_superior/documentos/ 2018/censo_da_educacao_superior_2017-notas_estatisticas2.pdf. Acessado: 16-09-2020.

[20] Pró-Reitoria de Graduação da UEFS. [n.d.]. Monitoria. http://www.prograd.uefs. $\mathrm{br} / \mathrm{modules} /$ conteudo/conteudo.php? conteudo $=23$

[21] Gustavo Coêlho de Oliveira, Fernanda Pereira de Souza, and Edineide Nunes da Silva. 2019. Papel da monitoria na formação acadêmica: um relato de experiência. Revista de Pesquisa Interdisciplinar 2, 2.0 (2019). 
[22] Luiz Fernandes Dourado and João Ferreira de Oliveira. 2009. A qualidade da educação: perspectivas e desafios. Cadernos Cedes 29, 78 (2009), 201-215.

[23] Paulo Freire. 2011. Pedagogia da autonomia: saberes necessários à pratica educativa (1st ed.). Paz e Terra.

[24] Lourdes Maria Bragagnolo Frison. 2016. Monitoria: uma modalidade de ensino que potencializa a aprendizagem colaborativa e autorregulada. Pro-Posições 27, 1 (2016), 133-153.

[25] Moacir Gadotti. 2010. Qualidade na educação: uma nova abordagem. Produção de terceiros sobre Paulo Freire; Série Livros (2010).

[26] Diulia Galvani, Bruna Corrêa Silva, Bruna Martins de Menezes, Diulia Zolin Galvani, and Patrícia Forgiarini Firpo. 2017. MONITORIAS EM PROJETO PEDAGÓGICO NO ENSINO SUPERIOR: UM RELATO DE EXPERIÊNCIA. Anais do Salão Internacional de Ensino, Pesquisa e Extensão 9, 1 (2017).

[27] Bernardete A Gatti. 2016. Formação de professores: condições e problemas atuais Revista internacional de formação de professores 1, 2 (2016), 161-171.

[28] Antonio Carlos Gil et al. 2002. Como elaborar projetos de pesquisa. Vol. 4. Atlas São Paulo.

[29] Elenilton Godoy and Eustáquio Almeida. 2017. A evasão nos cursos de Engenharia e a sua relação com a Matemática: uma análise a partir do COBENGE. Educação Matemática Debate 1, 3 (dec 2017), 339-361. https://doi.org/10.24116/ emd25266136v1n32017a05

[30] Hartmut Günther. 2006. Pesquisa qualitativa versus pesquisa quantitativa: esta é a questão? Psicologia: teoria e pesquisa 22, 2 (2006), 201-209.

[31] Mark J. Guzdial and Barbara Ericson. 2009. Introduction to Computing and Programming in Python, A Multimedia Approach (2nd ed.). Prentice Hall Press, USA.

[32] Leandro Fragoso Lins, Lucia Maia Cavalcanti Ferreira, Lucíola Vilarim Ferraz, and SSG de CARVALHO. 2009. A importância da monitoria na formação acadêmica do monitor. Fornada de ensino, pesquisa e extensão, IX (2009).

[33] Morgana Oliveira Lira, DQ Nascimento, GC de L Silva, and A dos S Maman. 2015. Contribuições da monitoria acadêmica para o processo de formação inicial docente de Licenciandos em Ciências Biológicas da UEPB. In II Congresso Nacional de Educação.

[34] Mário Rui Lourenço and Joaquim Machado. 2017. Aprender juntos: projeto de apoio curricular entre pares. Revista Portuguesa de Investigação Educacional 17 (2017), 124-145.

[35] Lívia Dourado Magalhães, Ionara de Souza Januário, and Anna Karla Fausto Maia. 2014. A monitoria acadêmica da disciplina de cuidados críticos para a enfermagem: um relato de experiência. Revista da Universidade Vale do Rio Verde 12,2 (2014), 556-565.

[36] Leonardo Magela Lopes Matoso. 2014. A importância da monitoria na formação acadêmica do monitor: um relato de experiência. CATUSSABA-ISSN 2237-3608 3 , 2 (2014), 77-83.

[37] Eric Mazur. 1999. Peer instruction: A user's manual.

[38] Vanderli Fava OLIVEIRA, Pedro L QUEIROS, Mario Neto BORGES, João Sérgio CORDEIRO, Marcia RF DIAS, LIMA Brito, AGUIAR Roldão Jr, G Benedito, Nival Nunes ALMEIDA, Paulo R SILVA, et al. 2010. Trajetória e estado da arte da formação em Engenharia, Arquitetura e Agronomia-volume I: Engenharias. Brasília: Instituto Nacional de Estudos e Pesquisas Educacionais Anísio Teixeira, Conselho Federal de Engenharia, Arquitetura e Agronomia (2010).

[39] Adriana Pelizzari, M de L Kriegl, Márcia Pirih Baron, Nelcy Teresinha Lubi Finck, and Solange Inês Dorocinski. 2002. Teoria da aprendizagem significativa segundo Ausubel. revista PEC 2, 1 (2002), 37-42.

[40] Fábio Inácio Pereira. 2017. APRENDIZAGEM POR PARES E OS DESAFIOS DA EDUCAÇÃO PARA O SENSO CRÍTICO. International fournal on Active Learning 2, 1 (2017), 6-12.

[41] Dilermando Piva Jr and Ricardo L Freitas. 2010. Estratégias para melhorar os processos de abstração na disciplina de Algoritmos. In Brazilian Symposium on Computers in Education (Simpósio Brasileiro de Informática na Educação-SBIE), Vol. 1.

[42] Vivian W REIS, Paulo JM Cunha, and IMPA Spritzer. 2012. Evasão no ensino superior de engenharia no Brasil: um estudo de caso no CEFET/RJ. In XL Congresso Brasileiro de Educação em Engenharia. Belém do Pará.

[43] Leila Ribeiro, Luciana Foss, and Simone André da Costa Cavalheiro. 2017. Entendendo o Pensamento Computacional. CoRR abs/1707.00338 (2017). arXiv:1707.00338 http://arxiv.org/abs/1707.00338

[44] Anthony Robins, Janet Rountree, and Nathan Rountree. 2003. Learning and Teaching Programming: A Review and Discussion. Computer Science Education 13, 2 (2003), 137-172. https://doi.org/10.1076/csed.13.2.137.14200 arXiv:https://doi.org/10.1076/csed.13.2.137.14200

[45] Bianca Santana, José Solenir Figuerêdo, and Roberto A. Bittencourt. 2017. Motivação de Estudantes Non-Majors em uma Disciplina de Programação. In Anais do XXV Workshop sobre Educação em Computação (São Paulo). SBC, Porto Alegre, RS, Brasil. https://doi.org/10.5753/wei.2017.3545

[46] Antônio Joaquim Severino. 2009. Expansão do ensino superior: contextos, desafios, possibilidades. Avaliação: Revista da Avaliação da Educação Superior (Campinas) 14, 2 (2009), 253-266.
[47] Edna Lúcia da Silva and Estera Muszkat Menezes. 2001. Metodologia da pesquisa e elaboração de dissertação. (2001).

[48] Eduardo Silveira and Fernanda de Sales. 2016. A importância do Programa de Monitoria no ensino de Biblioteconomia da Universidade do Estado de Santa Catarina (UDESC). InCID: Revista de Ciência da Informação e Documentação 7, 1 (2016), 131-149.

[49] Liane Margarida Rockenbach Tarouco, Letícia Coelho Roland, Marie-Christine Julie Mascarenhas Fabre, and Mary Lúcia Pedroso Konrath. 2004. Jogos educacionais. RENOTE: revista novas tecnologias na educação [recurso eletrônico] 2, 1 (2004).

[50] Romero Tavares. 2004. Aprendizagem significativa. Revista conceitos 10, 55 (2004).

[51] UEFS. 2010. Resolução CONSEPE Resolução CONSEPE 208/2010. Conselho Superior de Ensino, Pesquisa e Extensão - CONSEPE (2010).

[52] Clara Vasconcelos, João Félix Praia, and Leandro S Almeida. 2003. Teorias de aprendizagem e o ensino/aprendizagem das ciências: da instrução à aprendizagem. Psicologia escolar e educacional 7, 1 (2003), 11-19.

[53] Christopher Watson and Frederick W.B. Li. 2014. Failure Rates in Introductory Programming Revisited. In Proceedings of the 2014 Conference on Innovation Technology in Computer Science Education (Uppsala, Sweden) (ITiCSE '14). Association for Computing Machinery, New York, NY, USA, 39-44. https: //doi.org/10.1145/2591708.2591749

[54] Humberto Zanetti and Claudio Oliveira. 2015. Práticas de ensino de Programação de Computadores com Robótica Pedagógica e aplicação de Pensamento Computacional. In Anais dos Workshops do Congresso Brasileiro de Informática na Educação, Vol. 4. 1236. 